\title{
Aproximación critica sobre la transformación de la noción de desarrollo en el marco de la cooperación de España en Colombia
}

\section{Critical approach to the transformation of the development idea in the framework of Spanish cooperation towards Colombia}

\author{
Paola Méndez (iD
}

Politécnico Grancolombiano, Colombia

pmendez@poligran.edu.co

\section{César Niño iD}

Universidad Sergio Arboleda, Colombia

cesar.nino@usa.edu.co

\begin{abstract}
Resumen
La idea de desarrollo de la cooperación española en Colombia ha tenido grandes cambios en los últimos años. En este artículo se plantea el interrogante: ¿cómo se ha transformado la noción de desarrollo en la cooperación española en Colombia? De esta manera, con base en el análisis documental de fuentes oficiales y académicas, identificamos cuestiones fundamentales para ser abordadas desde una perspectiva crítica sobre los cambios conceptuales asociados al desarrollo en dicha cooperación. Se encuentra que la noción de desarrollo, en el contexto de la cooperación de España en Colombia, ha respondido a los cambios en la agenda internacional de desarrollo, es decir, empezó como una idea de lucha contra la pobreza, pero ha ido experimentando cambios asociados a los contextos particulares del país, desenclavando la perspectiva asociada a la seguridad de la agenda a asuntos como la ciencia, la construcción de la paz y del tejido social.
\end{abstract}

Palabras clave: noción de desarrollo, cooperación, Colombia, España.

\begin{abstract}
The idea of development in Spanish cooperation towards Colombia has undergone great changes in the recent years. Thus, we ask ourselves the question of how has the notion of development been transformed in Spanish cooperation towards Colombia? Based on the documentary analysis of official and academic sources, we identify fundamental questions to be approached from a critical perspective in the conceptual changes associated with development in Spanish cooperation towards Colombia. As such, we find that the notion of development within Spain's cooperation towards Colombia has responded to changes in the international development agenda, that is, it began as an embryonic idea of fighting poverty, but has been experiencing changes associated with the particular Colombian contexts unlocking the security perspective from the agenda such as science, peace building and restoration of the social fabric.
\end{abstract}

Keywords: Notion of development, cooperation, Colombia, Spain.

Articulo: Recibido el 16 de marzo de 2021 y aprobado el 07 de mayo de 2021

\section{Cómo citar este artículo:}

Mendez, P. \& Niño, C. (2021). Aproximación crítica sobre la transformación de la noción de desarrollo en el marco de la cooperación de España en Colombia. Reflexión politica 23(47), pp. 73-81. doi: https://doi.org/10.29375/01240781.4109 


\section{Introducción}

Las primeras dos décadas del siglo XXI han sido de grandes contrastes en materia de desarrollo para Colombia. La histórica relación bilateral con España ha estado inmersa en dichos contrastes que van desde la configuración de una idea castrense de seguridad y defensa, a la lucha contra el narcotráfico en 2001 (G. Molano, 2009), pasando por el trabajo conjunto entre Madrid y Bogotá en asuntos de paz territorial, cambio climático e inclusión de comunidades marginadas en proyectos propuestos entre 2018 y 2021. Así las cosas, Colombia se fue convirtiendo en un punto focal de la idea de la iberoamericanidad que tuvo un gran impulso entrados los años ochenta del siglo pasado (Menchero \& Ríos, 2020).

Sin embargo, es pertinente revisar la noción de desarrollo que se ha construido, la cual se ha transformado en la agenda de cooperación española en Colombia. En los últimos 20 años, los cambios sociales, los fenómenos políticos y las configuraciones territoriales en el país han llevado a la creación de una constelación de elementos que son sujetos de profundos análisis. Por esa razón, el centro de gravedad del presente artículo está en la noción de desarrollo que se encuentra dentro del marco de la cooperación española hacia Colombia. De tal manera, en primer lugar, se aborda una propuesta metodológica con base en la evidencia sobre los documentos oficiales, planes y marcos de los últimos 20 años. Allí se expone la pregunta de investigación y la hipótesis por trabajar.

En segundo lugar, se presenta un marco analítico sobre la noción de desarrollo. Se hace una aproximación desde una perspectiva crítica en la que se enfatiza en diferentes cuestionamientos como: ¿qué entiende España por desarrollo?, ¿qué es desarrollo?, ¿cuándo se es desarrollado? y ¿qué viene después del desarrollo?, entre otras.

En tercer lugar, se hace un análisis con base en los sectores de aplicación de la cooperación española en Colombia, se usan los Planes Directores y el Marco de Asociación País para detectar la transformación de la noción de desarrollo en dicho contexto. Finalmente, proponemos una serie de conclusiones que son fundamentales para continuar con futuras investigaciones sobre el desarrollo y, en particular, la cooperación española en Colombia.

\section{Metodologia}

La investigación se realizó con base en el análisis documental de fuentes oficiales y académicas. Lo anterior permitió identificar las cuestiones fundamentales para ser abordadas desde una perspectiva crítica sobre los cambios conceptuales asociados al desarrollo en el contexto de la cooperación española en Colombia. Bajo dicha lógica, fue necesario rastrear las estrategias de desarrollo de España entre 1964 y 1975, allí se encontraron arquitecturas deshumanizantes del modelo desarrollista que, con la transición a la democracia, se sintonizaron con las demandas europeas y con la apertura del desarrollo asociado a cuestiones sociales estructurales.

Por otra parte, se hizo una aproximación longitudinal tomando como insumos los Planes Directores de la Cooperación Española (PDCE) desde 2001 hasta 2021. Dichos PDCE sirvieron para dar cuenta de los ajustes narrativos asociados al desarrollo en los últimos 20 años desde España. También se elaboró un análisis sobre los sectores de aplicación de la cooperación en Colombia y sobre el Marco de Asociación País España-Colombia 2015-2019 (MAP). De esta manera, se hizo un cruce entre los PDCE, el MAP y los sectores de aplicación.

En efecto, la pregunta que guía la presente investigación es: ¿cómo se ha transformado la noción de desarrollo en la cooperación española en Colombia? Con base en lo anterior, la hipótesis por comprobar es que la noción de desarrollo de la cooperación de España en Colombia ha respondido a los cambios en la agenda internacional de desarrollo. Es decir, empezó como una idea de lucha contra la pobreza, pero ha ido experimentando cambios asociados a los contextos particulares colombianos, por ejemplo, dinámicas técnicas, científicas y combate contra el narcotráfico, hasta la complejidad territorial en materia de implementación del Acuerdo de Paz. Merece la pena mencionar que la transformación del concepto 
de desarrollo ha tenido dilemas entre la ayuda hacia un país con graves problemas estructurales y atisbos de país de renta media enclavado en la Organización para la Cooperación y el Desarrollo Económicos (OCDE).

\section{Marco analitico: ¿qué entiende España por desarrollo?}

El desarrollo como concepto aparece a finales de los años cuarenta, cuando las tensiones y complejidades de la Segunda Guerra Mundial terminaron y cedieron terreno a otros asuntos en la agenda internacional. Dentro del marco de la disputa ideológica, política y económica que sucedió a la emergencia de la Guerra Fría, los tomadores de decisiones, sobre todo del bloque occidental, se concentraron en el fenómeno de la pobreza. No por su novedad, sino por lo determinante que resultaba en un contexto de disyuntivas entre modelos de Estado y de proyectos económicos a escalas nacionales.

En 1949, el discurso de posesión del presidente estadounidense Harry Truman (19451953) dio inicio simbólico a una era del desarrollo cuya preocupación vertebral era el subdesarrollo y los problemas derivados del mismo. En ese contexto, Washington identificó la existencia de áreas no avanzadas en el mundo y autoproclamó su misión de llevar la modernidad a aquellos sitios que carecían de esta, difundiendo políticas idóneas para lograr tal situación (Escobar, 2007). El subdesarrollo era, entonces, comprendido como una consecuencia de medidas económicas erradas y un estadio anterior al desarrollo que, para ser alcanzado, debía surtir fases y etapas progresivas que desembocarían en la industrialización y en la urbanización. Desde entonces se gestó un imaginario del desarrollo como sinónimo de crecimiento económico y de valores occidentales como la democracia, el progreso, la libertad y los derechos, aun cuando las coyunturas de las décadas posteriores obligaron a la incorporación de perspectivas sociales y ambientales.

Para superar esas políticas erradas a las que se atribuía el subdesarrollo hubo misiones de expertos y de las nacientes organizaciones internacionales de la época, para diagnosticar lo que fallaba en las regiones pobres en términos de políticas públicas y, en general, de la organización política y administrativa (Escobar, 2007). Los países latinoamericanos, que -junto a algunos africanos y asiáticos- experimentaron la implementación de fórmulas importadas para superar el subdesarrollo, ofrecieron lecturas divergentes asociadas a este fenómeno (Stiglitz, 2002). Desde la región surgieron corrientes teóricas que consideraban que el subdesarrollo no obedecía a enfoques de política económica errados, sino a causas externas producto de los sistemas comerciales internacionales que configuraban una estructura de centro y periferia con términos de intercambio asimétricos entre sí.

En otras palabras, desarrollo y subdesarrollo coexistían de manera simultánea y no se trataba de una lectura de etapas progresivas (Escobar, 2007; Niño \& Méndez, 2019). Uno de los ejes problemáticos, a la luz de esas perspectivas, era la brecha tecnológica entre los países del centro y los de la periferia, por lo que se propuso el Modelo de Industrialización por Sustitución de Importaciones (ISI). Este suponía, entre otras cosas, el avance tecnológico y la manufactura local de los bienes que hasta entonces se importaban, despertando además una lógica de oferta y demanda nacional que dinamizara la economía. Si bien este experimento se llevó a cabo por más de una década en varios países de la región, como México, Argentina, Chile y Brasil, también generó problemas económicos profundos pues no se contempló la exportación de los excedentes de la producción y el endeudamiento desbordó las capacidades de los países. A grandes rasgos, el resultado de los avances tecnológicos se vio deslegitimado por la crisis económica en la que incurrieron los países latinoamericanos, a tal punto que se hace referencia histórica a los años ochenta como la década pérdida de la región. A partir del mismo periodo, y en contraposición a la tendencia que había surgido en América Latina, se posiciona la propuesta neoliberal en la que se desvirtúa el rol del Estado en los asuntos económicos, se defiende el mercado como mecanismo ideal y se insta a un proceso de liberalización en el marco del comercio internacional.

De manera simultánea ebullian otras cuestiones globales de naturaleza social y ambiental. A partir de los años setenta, de cara a la promesa incumplida del desarrollo, las preocupaciones por el bienestar humano y el estado del medio ambiente físico cobraron protagonismo en una agenda que había relegado temas semejantes en las décadas anteriores. La academia desempeñó un rol fundamental que, a modo de advertencia, 
puso en conocimiento el deterioro de los sistemas naturales y el peligro que eso suponía para la vida en su forma conocida. La sociedad civil, por su parte, también participó en la difusión de una preocupación cada vez más apremiante, por medio de la creación de organizaciones ciudadanas y de colectivos de activismo en general.

En la esfera política, en cambio, el tema ambiental fue relativamente ajeno hasta que informes como Los límites del crecimiento, construido por el MIT y socializado en el seno del Club de Roma en 1972, permitió su escalamiento a los círculos de tomadores de decisiones. Este escenario se configuró como un foro internacional para discutir desafíos globales. Su mencionado informe fue percibido como una lectura radical pues, como su nombre lo indica, sugería la imposibilidad de un crecimiento ilimitado. Esta perspectiva despertó incomodidades que la Declaración de Estocolmo 1972 morigeró con avidez para llegar a instancias de convergencia política y diplomática alrededor del asunto medioambiental. Este último referente apostó por una postura más neutral, que consideraba la existencia de recursos limitados, pero reconocía la importancia de crecer para alcanzar estadios de desarrollo. En otras palabras, se trataba de una interpretación del crecimiento que manera impajaritable, debía contemplar la importancia de un medioambiente limitado en recursos.

La Declaración de Estocolmo antecedió la definición de un concepto vertebral para el desarrollo a partir de los años ochenta, a saber, el de desarrollo sostenible. Este se configuró a partir del Informe Brundtland en 1987, que sostuvo un enfoque mediante el cual se deben satisfacer las necesidades presentes sin comprometer a las generaciones venideras y sus respectivas demandas (Comisión Mundial sobre el Medio Ambiente y el Desarrollo, 1987). Si bien no es la única definición existente, se trató de una aproximación pionera que sigue siendo referente. Desde entonces, la búsqueda de armonía entre las dimensiones económica, ambiental y social es pilar de la noción de desarrollo. Ahora bien, desde el punto de vista del concepto de desarrollo, la dimensión social atravesaba coyunturas particulares propias que provocaron su asentamiento definitivo en la agenda del desarrollo.

Las preocupaciones por el bienestar humano también se acrecentaron y se tornaron más complejas en la década de los setenta, de cara al fenómeno de la pobreza. Las coyunturas dieron paso a un cambio paradigmático en el que, en el seno de la academia y los movimientos de la sociedad civil, se otorgó protagonismo a la satisfacción de necesidades por encima de la acumulación material. Se trató de lecturas alternativas al desarrollo en las que el ser humano se configuró como eje, en tanto sujeto capaz que pretende la expansión de oportunidades para el bienestar de su vida desde la dignidad. Esa perspectiva fue en gran medida recopilada y consolidada por la noción de desarrollo humano como corriente, que de alguna manera centraba el foco en la pregunta por el desarrollo para quién.

La confluencia de preocupaciones sociales y ambientales derivó en una visión del desarrollo que, desde los años noventa, no ha sido reversible. La triada de asuntos económicos, pero también sociales y ambientales, ha configurado una agenda del desarrollo diversa y con múltiples temáticas, dada la complejidad de fenómenos que puede incorporar. De lo anterior es muestra la Agenda del Milenio o los Objetivos de Desarrollo del Milenio (ODM), que orientaron perspectivas de desarrollo entre los años 2000 y 2015. Estos compilaron un conjunto de ocho propósitos ambiciosos, aunque solo fueron pensados para países en vía de desarrollo, lo que supuso incomodidades en la medida que no se perseguían de manera universal y en los mismos términos. Cuando llegó 2015 y los ODM caducaron, la comunidad internacional fue testigo de objetivos sin cumplir y más tareas pendientes que problemas resueltos. Las lecciones aprendidas se tuvieron en cuenta en la agenda que les sucedió, a saber, la 2030 o los Objetivos de Desarrollo Sostenible (ODS), prevista para el periodo 2015-2030. Este proyecto fue aún más ambicioso (17 objetivos), mucho más participativo, garantizó mejor financiación que los ODM y, sobre todo, incluyó a todos los países como parte de los objetivos por cumplir (Sotillo, 2015). En otras palabras, se trata de una agenda global vigente, en la que cada país se configura como responsable del cumplimiento de los objetivos y, por ende, reconoce problemas urgentes por atender.

Con matices entre unos países y otros, los escenarios nacionales e internacionales han acogido perspectivas sobre el desarrollo que versan sobre lo anterior y -aunque no de manera exclusiva, porque hay perspectivas alternativas $y$ 
críticas radicalmente diferentes-, han orientado la toma de decisiones. La cooperación internacional, por ejemplo, no ha sido ajena a esa dinámica y a las transformaciones de la agenda del desarrollo.

No obstante, es menester reconocer que, en la práctica, perviven nociones de desarrollo que tácitamente priorizan los temas económicos y, por ende, confunden la idea moderna de desarrollo con la de crecimiento. Entonces, como es notorio, las discusiones sobre ese concepto han experimentado tensiones entre la visión más rígida de los indicadores y la idea de plasticidad en la humanización de las estadísticas. Esto significa que hay una noción enclavada en dinámicas económicas y de modelo político que distorsionan una apropiada lectura integral del desarrollo.

Ahora bien, aterrizando la discusión sobre el desarrollo al caso español, este país lo concibe, dentro de su programa de cooperación, como un objetivo dentro del marco de la gobernanza global del desarrollo, es decir que lo asume como un derrotero asociado al progreso económico y social global, sostenible, inclusivo y equitativo, que busque la justicia y la igualdad ciudadana (Cooperación Española, s. f.). En esa noción se encuentran elementos que dan cuenta de la transformación global del desarrollo. Sin embargo, el recorrido de ese concepto no siempre ha contado con tal diversidad.

La época de 1964 a 1975 fue para España un periodo de planificación indicativa, para el cual el objetivo de desarrollo fue crucial. Este fue entendido como crecimiento de la renta nacional y estuvo inclinado a favor del sector industrial al margen de transformaciones en términos institucionales o sociales, cuyo protagonismo fue secundario (Alsina-Oliva, 1987). La posterior transición a la democracia experimentó el brote de los problemas estructurales que la planificación había dejado pese al crecimiento que había provocado, y puso en evidencia las situaciones que no se habían atendido de manera oportuna. Sin embargo, también fue un proceso que, de cara a la apertura democrática, dio lugar a una agenda de preocupaciones más amplia y diversa, consecuente con el ingreso de España a la Comunidad Europea. En otras palabras, se configuró un proceso de modernización social que, entre otros asuntos, considera la consolidación del Estado de bienestar y la reducción de las desigualdades sociales (Cabrejas de las Heras, 2004).
La apertura temática en relación con la noción de desarrollo también se empezó a ver reflejada en la complejidad de la cooperación española con América Latina a partir de los años ochenta, en la medida en que dio cuenta de una multiplicidad de asuntos en las agendas bilaterales y multilaterales como parte de una idea amplia del desarrollo. Desde entonces se acogen cuestiones que desbordan lo económico e incluyen fenómenos o situaciones que hacen parte de las esferas social, cultural y ambiental.

\section{Aplicación de la noción española de desarrollo en Colombia}

En efecto, como se analizó en el apartado anterior, los diseños de los marcos analíticos en materia de desarrollo reflejan en algunas oportunidades ciertos acaparamientos de las ideas economicistas, que comprenden lecturas deshumanizantes. Aquellas ideas son enclaves $\mathrm{y}$ herencias del siglo XX y, por supuesto, de las arquitecturas y los modelos de los consensos de Washington y Beijing (Li, 2015; Sanahuja y Schünemann, 2012; The Economist, 2017) por mencionar algunos marcos dominantes.

En la perspectiva que concierne a Colombia como país receptor de la cooperación al desarrollo, Bogotá se convirtió en un escenario complejo y polivalente. Complejo porque el país ha tenido un inicio de siglo XXI con buenos indicadores macroeconómicos, pero con un profundo desastre en lo concerniente al Índice de Desarrollo Humano (IDH) (Programa de Las Naciones Unidas para el Desarrollo, 2016), y polivalente porque distintos actores internacionales han visto a Bogotá como ventana de oportunidad para diversos escenarios que ellos mismos han construido como idea de desarrollo. En especial, lo concerniente a la seguridad nacional, la lucha contra el terrorismo y el narcotráfico, así como la construcción de una agenda rígida asociada a elementos de políticas públicas tradicionales (A. Molano, 2015; Montero, 2017). Dentro de dichas complejidades y polivalencias, desde una óptica europea, los temas sobre la paz territorial, la construcción de agendas de negociación, la sustitución de cultivos (Garzón et al., 2019) y el acompañamiento a la construcción de la paz ha sido un derrotero interesante asociado al desarrollo. 
Las relaciones en materia de cooperación entre España y Colombia tienen unas profundas raíces en cuestiones científicas y técnicas desde 1979 con el Convenio Básico de Cooperación Científica y Técnica, así como también con el Acuerdo Complementario General de Cooperación del Convenio Básico de Cooperación Técnica y Científica de 1988 (Menchero \& Ríos, 2020). A simple vista es fácil detectar que los inicios de esta relación, al menos en el siglo XX, estuvieron cifrados por dinámicas distintas a los rígidos panoramas economicistas y securitarios que describen la relación entre Colombia y otros países.

En ese orden de ideas, el proceso de cambio cultural en la época posfranquista trajo consigo aproximaciones complejas con Colombia. La idea de resignificar la presencia española en Bogotá motivó una amplitud en distintos frentes (Duarte Herrera et al., 2017), entre ellos la noción de desarrollo que motivaría el inicio del nuevo siglo y con ella, incluso una apuesta transformativa geopolítica (Cairo, 2010), económica (Castrillón, 2016) y social (Matutes, 1996).

En 2001, España construyó el PDCE 20012004 en el que arrojó prospectivas de desarrollo asociadas al crecimiento económico. Así, identificó una narrativa en la que definió que, para 2020, los países en desarrollo y las economías en transición representarian el $60 \%$ del producto interno bruto (PIB) mundial (Gobierno de España, 2001). Allí Madrid definió tres principales ejes como caminos al desarrollo: la inversión pública y privada, el comercio libre y la Ayuda Oficial al Desarrollo (AOD).

En efecto, el PDCE 2001-2004 distingue, entre los países prioritarios para la cooperación, los países en conflicto o en reconstrucción/ posconflicto con dos grandes bloques formados por Colombia y los Balcanes. De esa forma, como objetivo se pretende que los países de concentración preferente sean destinatarios, en conjunto, de más de 125.119 millones de pesetas solo en concepto de ayuda bilateral para 2004 (Gobierno de España, 2001). Mientras esto sucedía, Colombia atravesaba por una de las etapas más difíciles de la historia de su conflicto armado, razón que empujó a que la relación entre Bogotá y Washington se estrechara, lo que arrojó como resultado la arquitectura y puesta en marcha del Plan Colombia. Este plan contrastó con la sencilla idea de desarrollo asociada al progreso económico y la lucha contra el narcotráfico que trajo consigo una perspectiva securitaria tanto de la cooperación como del desarrollo (Ríos \& Gago, 2018). Así, mientras desde Washington se definía una narrativa securitaria del desarrollo en la cooperación para Colombia, Madrid configuró a Bogotá como objeto de un programa dirigido al acompañamiento del arreglo del conflicto.

Por otra parte, en el PDCE 2005-2008 España alcanza a ampliar la agenda y le da paso a sus prioridades horizontales ligadas a: lucha contra la pobreza, defensa de los derechos humanos, equidad de género, sostenibilidad medioambiental y diversidad cultural (Gobierno de España, 2008). Lo anterior se sincroniza con la noción de desarrollo trabajada inicialmente, que compatibiliza con la propuesta de gobernanza global del desarrollo. Así, dentro de dichas prioridades horizontales, Colombia pasa de ser un país "prioritario" a ser un país con "atención especial". Dicha categoría alcanzó un significado importante debido a que su referencia hace hincapié en que "lo especial" se asocia con la necesidad de prevenir y resolver los conflictos, la construcción de paz, la debilidad institucional, y factores tanto naturales como del sistema político que agravan la pobreza y agudizan la violencia. Colombia ocupó dicha clasificación junto con Cuba, Irak, Líbano, Siria, República Democrática del Congo, Etiopía, Guinea Ecuatorial, Sudán, Guinea Bissau, Timor Oriental, Afganistán, Camboya, Bosnia y Herzegovina, y Albania (Gobierno de España, 2008).

En ese orden de ideas, en el PDCE 2009-2012 Madrid profundiza las prioridades horizontales del Plan Director anterior en su noción de desarrollo. Construye una agenda más amplia, pero con mayor precisión en asuntos de migración, de construcción de paz, educación y gobernabilidad. En efecto, su estrategia tuvo áreas de acción en infancia y juventud, pueblos indígenas y población afrodescendiente. Lo anterior significa que España hizo una lectura contextual y compleja sobre algunas particularidades y en esta oportunidad, dentro del PDCE, Colombia estuvo clasificada en el grupo de "atención focalizada" (Gobierno de España, 2009).

Según la narrativa española, aquella "atención focalizada" incluye a Colombia por su potencial como socio de desarrollo, en el que se identifican particularidades precisas e instrumentos selectivos de aplicación asociados al conflicto y a la construcción de la paz (Gutiérrez, 2015; Medina, 2019; Ríos et al., 2013). Explica que, 
como inferencia causal, la resolución del conflicto armado puede llevar a que los demás sectores se potencien y evolucionen, y si dicho conflicto se perpetúa, los problemas en las demás áreas priorizadas se deterioran y erosionan.

En el PDCE 2013-2016, Colombia se encuentra dentro del marco de "país socio". Así, Madrid definió que la relación se configura de acuerdo con el contexto regional y nacional. Desde España se concibió que la existencia del conflicto armado permitía un mayor compromiso sostenido que alcanza a justificar el alcance y la viabilidad de su actividad en el territorio colombiano. Se pone de manifiesto que los asuntos que lleven a la construcción de la paz serán atendidos con especial atención porque impactan distintas áreas del desarrollo, entre ellas, el medio ambiente, las poblaciones étnicas, la cultura (Menchero \& Ríos, 2020) y el Estado social de derecho (Gobierno de España, 2013).

Con base en lo anterior, la construcción del MAP 2015-2019 se complementa con los planes directores del momento. Dicho marco se traza los siguientes retos (gráfico 1):

Grafico 1. Principales retos 2015-2019 en Colombia

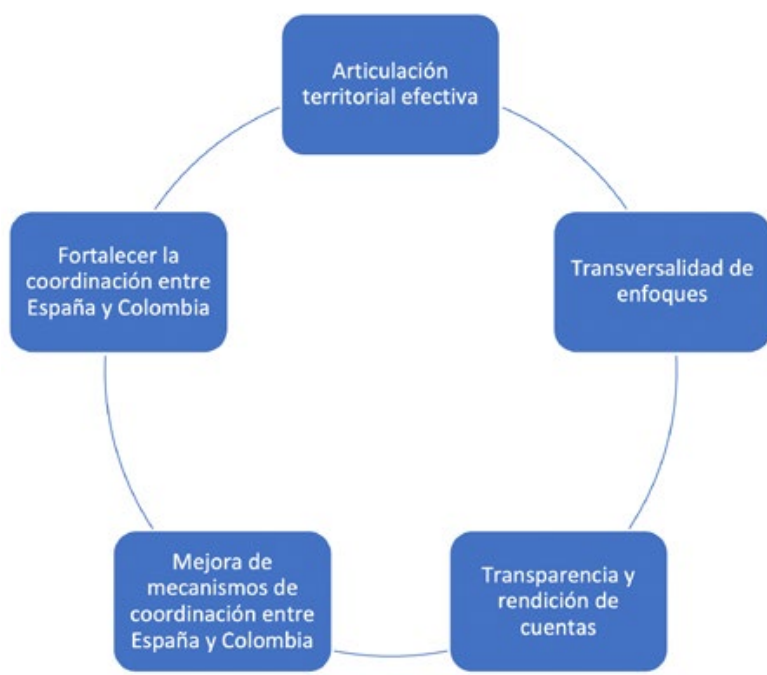

Fuente: elaboración propia con base en Cooperación Española, 2015.

El MAP explica cómo ha sido el proceso de cooperación al desarrollo en Colombia. Es interesante observar que ha habido una evolución y ampliación de los conceptos tanto de cooperación como de desarrollo. De hecho, el documento hace un balance importante sobre la participación española, en el que se enfatiza que en Colombia hay una multiplicidad de actores de la Cooperación
Española. Por ejemplo, se señala que diez ministerios españoles han apoyado actuaciones de cooperación en Colombia en el periodo 2011-2014, por otro lado, han estado presentes 14 comunidades autónomas, 76 entidades locales (ayuntamientos y diputaciones), y 32 universidades han tenido acciones focalizadas en materia de investigación, ciencia y desarrollo (Cooperación Española, 2015, p. 24).

Por otro lado, en el PDCE 2018-2021, el gobierno español ha incluido a Colombia en el grupo de países de renta media. En efecto, dicha categoría significa que la ayuda al desarrollo que recibe no supone una aportación absoluta de gran magnitud en relación con la renta nacional bruta (Gobierno de España, 2017). En esta oportunidad, entre los años 2018-2021, el centro de gravedad en la noción de desarrollo está determinado por la construcción de resiliencia, el apoyo en su transición hacia modelos productivos sostenibles, la garantía de servicios sociales de calidad, el fomento de la diversidad y la inclusión, y la construcción institucional (Gobierno de España, 2017, p. 48) (gráfico 2).

Grafico 2. Menciones sobre Colombia en los Planes Directores 2001-2021

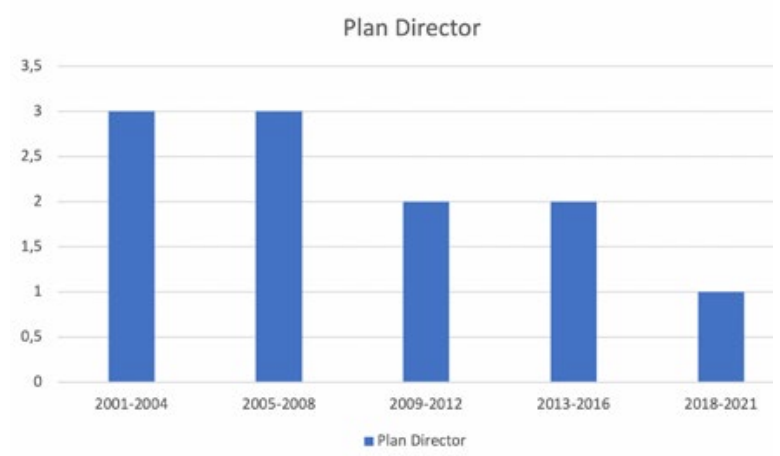

Fuente: elaboración propia con base en (Gobierno de España, 2001, 2008, 2009, 2013, 2017).

De esta manera, si bien las menciones sobre Colombia en los PDCE desde 2001 hasta 2021 no han sido las protagonistas de la hoja de ruta para Iberoamérica, es evidente que el inicio del siglo XXI estuvo marcado por grandes sucesos en la coyuntura colombiana que llamaron la atención de Madrid. Sin embargo, en términos macros, las menciones fueron disminuyendo a medida que los nuevos planes se desarrollaban y las transformaciones sobre la idea de desarrollo aplicado a Colombia se acomodaban a dimensiones más estructurales que la noción de progreso económico (Castrillón, 2016). 


\section{Conclusiones}

El dinamismo de los fenómenos, las circunstancias particulares de la gobernanza global y las cuestiones asimétricas de las relaciones internacionales han logrado identificar que la noción del desarrollo responde a poliedros y lentes de análisis diferentes. Es decir, no hay una única respuesta ni una única visión válida del concepto. Sin embargo, sí es claro que dicha idea debe responder a lógicas integrales y no exclusivamente económicas.

Uno de los principales hallazgos es que la apertura democrática española produjo un cambio paradigmático en el concepto de desarrollo que lo tornó más amplio, complejo e inclusivo. Esto significa, entonces, que es mucho más fácil identificar transformaciones sobre el desarrollo con cambios de régimen político. No obstante, para el caso colombiano, es más complejo determinar su propia noción de desarrollo teniendo en cuenta que su contexto no tiene que ver con cambio de régimen, sino con lógicas de conflicto a posconflicto. Una realidad que ha logrado comprender Madrid para la proyección de su dinámico proceso de idea de desarrollo.

En el presente artículo, la idea de desarrollo que es centro de gravedad en la relación entre España y Colombia ha sido cambiante. En otras palabras, ha respondido a los cambios en la agenda internacional de desarrollo. Es decir, empezó como una idea de lucha contra la pobreza, pero ha ido experimentando cambios asociados a los contextos particulares del país. Lo anterior es muestra de una política exterior española con claros y definidos intereses en Colombia. Significa que, desde Madrid, ha habido una lectura estratégica y dinámica acorde a los acontecimientos colombianos de las últimas décadas.

En efecto, a diferencia de otros actores y agendas de cooperación para el desarrollo en Colombia, que centraron sus esfuerzos técnicos y diplomáticos en ecuaciones asociadas a la pobreza, el narcotráfico y el terrorismo, el plan español ha sostenido un menú de complejos capítulos. Aquellos se suscriben más a una cooperación para el desarrollo desde flancos humanitarios, tejidos sociales, construcción de paz y apoyo a la implementación del acuerdo entre las extintas Fuerzas Armadas Revolucionarias de Colombia (FARC) y el Estado. En otras palabras, España ha logrado desenclavar el tema securitario como eje exclusivo de la cooperación y el desarrollo en Colombia.

De esta manera, una lectura crítica sobre esta relación tiene que ver, entre otras, con la forma como se llevará a cabo dicho diálogo sobre el desarrollo entre España y Colombia teniendo en cuenta que este último país ingresó formalmente a la OCDE en 2018. Esto significa, entonces, que la idea de desarrollo desde España hacia Colombia tiene que seguir transformándose, así como la noción y autonoción de desarrollo de Colombia.

\section{Referencias}

Alsina-Oliva, R. (1987). Estrategia de desarrollo en España 1964-1975: planes y realidad. Cuadernos de economía: Spanish Journal of Economics and Finance, 15(44), 337-370.

Cabrejas de las Heras, G. (2004). Transformación de la sociedad española desde 1970: cambios y permanencias en la institución familiar. En La transición a la democracia en España (p. 31). Confederación de Asociaciones de Archiveros, Bibliotecarios, Museólogos y Documentalistas, ANABAD, Asociación de Amigos del Archivo Histórico Provincial de Castilla La Mancha.

Cairo, H. (2010). El pivote geográfico de la historia”, el surgimiento de la geopolítica clásica y la persistencia de una interpretación telúrica de la política global. Geopolitica(s), 1(2), 321-331.

Castrillón, J. H. (2016). Cooperación para el desarrollo España-Colombia, un enfoque de economía política. Universidad Complutense de Madrid.

Comisión Mundial sobre el Medio Ambiente y el Desarrollo. (1987). Informe de la Comisión Mundial sobre el Medio Ambiente y el Desarrollo "Nuestro futuro común".

Cooperación Española. (s. f.). Entender la cooperación. Recuperado 22 de diciembre de 2020, de https:// cooperacionespanola.es/es/entender-la-cooperacion

Cooperación Española. (2015). Marco de Asociación País Colombia-España 2015-2019.

Duarte Herrera, L. K., González Parias, C. H., Mesa Bedoya, J. C., Duarte Herrera, L. K., González Parias, C. H., \& Mesa Bedoya, J. C. (2017). Challenges of International Cooperation for Development in Colombia. Estudios internacionales (Santiago), 49(188), 111-146. https://scielo.conicyt.cl/scielo.php?script=sci_arttext $\&$ pid $=$ S0719-37692017000400111\&lng=en\&n$\underline{\mathrm{rm}=\mathrm{iso} \& \mathrm{t} \operatorname{lng}=\mathrm{en}}$

Escobar, A. (2007). La invención del Tercer Mundo (D. Reyes (ed.); 1.a ed.). Fundación Editorial el perro y la rana. 
Garzón, J., Gélvez, J., \& Bernal, J. (2019). ¿En qué va la sustitución de cultivos ilícitos? Desafios, dilemas actuales y la urgencia de un consenso.

Gobierno de España. (2001). Plan Director de la Cooperación Española 2001-2004.

Gobierno de España. (2008). Plan Director de la Cooperación Española 2005-2008.

Gobierno de España. (2009). Plan Director de la Cooperación Española 2009-2012.

Gobierno de España. (2013). Plan director de la Cooperación Española 2013-2016.

Gobierno de España. (2017). Plan Director de la Cooperación Española 2018-2021.

Gutiérrez, F. (2015). “¿Una historia simple?” En Contribución al entendimiento del conflicto armado en Colombia (pp. 1-43). Comisión Histórica del Conflicto y sus Víctimas.

Li, H. (2015). The Chinese Model of Development and Its Implications The Chinese Model of Development and Its Implications. World Journal of Social Science Research, 2(2), 128-138

Matutes, A. (1996). 10 años de España en la Unión Europea. Objetivos de la política exterior española. CIDOB d'afers Internacionals, 34-35, 191-196.

Medina, M. (2019). La larga historia de violencia y resistencia en el Cauca. Razón Pública. https://www. razonpublica.com/index.php/conflicto-drogas-y-paz-temas-30/12423-la-larga-historia-de-violencia-y-resistencia-en-el-cauca.html

Menchero, M., \& Ríos, J. (2020). Programa Patrimonio Cultural y Escuelas Taller: análisis de la cooperación cultural española en América Latina. Aportes, Revista de Historia Contemporánea, 35(102), 149-183.

Molano, A. (2015). Justicia para el posconflicto: viejos y nuevos problemas en escenarios complejos. En El posconflicto en Colombia: reflexiones y propuestas para recorrer la transición (pp. 15-25). Instituto de Ciencia Política; Fundación Konrad Adenauer.

Molano, G. (2009). Cooperación para el combate ilícito de estupefacientes. Análisis Político, 66, 100-122.

Montero, A. (2017). El proceso de "desecuritización” en el conflicto armado colombiano para la construcción de la paz. Revista Ciudad Paz-ando, 10(1), 33-46.

Niño, C., \& Méndez, P. (2019). La noción china del desarrollo: la trampa de los indicadores. Papel Político, 24(2). https://doi.org/10.11144/Javeriana.papo24-2. ncdt

Programa de Las Naciones Unidas para el Desarrollo. (2016). Informe sobre Desarrollo Humano 2016. http://hdr.undp.org/sites/default/files/HDR2016 SP Overview_Web.pdf
Ríos, J., Bula, G., \& Brocate, R. (2013). Estado, Estado de Derecho y violencia armada en Colombia (2000-2011). Revista de Paz y Conflictos, 6(0), 6-31. https://doi.org/10.30827/revpaz.v6i0.427

Ríos, J., \& Gago, E. (2018). Realidades y desafíos de la paz territorial en Colombia. Papers. Revista de Sociologia, 103(2), 281. https://doi.org/10.5565/rev/ papers. 2361

Sotillo, J. Á. (2015). Los Objetivos de Desarrollo Sostenible. En El reto de cambiar el mundo. La Agenda 2030 de desarrollo sostenible (1.a ed., pp. 7895). Catarata.

Stiglitz, J. (2002). El malestar en la Globalización. Solar. 